Bull. Korean Math. Soc. 51 (2014), No. 6, pp. 1749-1771

http://dx.doi.org/10.4134/BKMS.2014.51.6.1749

\title{
ON SLANT RIEMANNIAN SUBMERSIONS FOR COSYMPLECTIC MANIFOLDS
}

\author{
İrem Küpeli ERken and Cengizhan Murathan
}

\begin{abstract}
In this paper, we introduce slant Riemannian submersions from cosymplectic manifolds onto Riemannian manifolds. We obtain some results on slant Riemannian submersions of a cosymplectic manifold. We also give examples and inequalities between the scalar curvature and squared mean curvature of fibres of such slant submersions in the cases where the characteristic vector field is vertical or horizontal.
\end{abstract}

\section{Introduction}

Riemannian submersions were introduced in the sixties by B. O'Neill and A. Gray (see [9], [20]) as a tool to study the geometry of a Riemannian manifold with an additional structure in terms of certain components, that is, the fibers and the base space. The Riemannian submersions are of great interest both in mathematics and physics, owing to their applications in the Yang-Mills theory ([3], [30]), Kaluza-Klein theory ([4], [12]), supergravity and superstring theories ([13], [18]), etc. Riemannian submersions were considered between almost complex manifolds by Watson in [29] under the name of almost Hermitian submersion. For Riemannian submersions between almost contact manifolds, Chinea [7] studied under the name of almost contact submersions. Further, B. Şahin [24] introduced a kind of submersion which was defined from almost Hermitian manifolds to any Riemannian manifolds. Recently there are several kinds of submersions according to the conditions on it: e.g., contact-complex submersion [10], quaternionic submersion [11], almost $h$-slant submersion and $h$-slant submersion [22], semi-invariant submersion [27], $h$-semi-invariant submersion [23], etc.

On the other hand, the study of slant submanifolds was initiated by B. Y. Chen in [6]. In [24], B. Şahin studied slant submersions from an almost Hermitian manifold to a Riemannian manifold and generalized his results which

Received November 15, 2013; Revised May 16, 2014.

2010 Mathematics Subject Classification. Primary 53C43, 53C55; Secondary 53D15.

Key words and phrases. Riemannian submersion, cosymplectic manifold, slant submersion.

This paper is supported by Uludag University research project (KUAP(F)-2012/57). 
were given in [25]. He also suggested to investigate slant submersions from almost contact metric manifolds onto Riemannian manifolds [26].

In this paper, we consider slant Riemannian submersions from cosymplectic manifolds. We obtain some results on slant Riemannian submersions of a cosymplectic manifolds.

The paper is organized in the following way. In Section 2, we recall some notions needed for this paper. Section 3 deals with cosymplectic manifolds. In Section 4, we give definition of slant Riemannian submersions and introduce slant Riemannian submersions from cosymplectic manifolds onto Riemannian manifolds. We survey main results of slant submersions defined on cosymplectic manifolds and obtain some interesting properties about them. We construct examples of slant submersions in the cases where the characteristic vector field $\xi$ is vertical or horizontal. We give a sufficient condition for a slant Riemannian submersion from cosymplectic manifolds onto Riemannian manifolds to be harmonic. Moreover, we investigate the geometry of leaves of ( $\operatorname{ker} F_{*}$ ) and $\left(\operatorname{ker} F_{*}\right)^{\perp}$. Here, we find a necessary and sufficient condition for a slant Riemannian submersion to be totally geodesic. We give sharp inequalities between the scalar curvature and squared mean curvature of fibres such that characteristic vector field $\xi$ is vertical or horizontal. Moreover, we know that the antiinvariant submersions are special slant submersions with slant angle $\theta=\frac{\pi}{2}$. We investigated such a submersions in [17]. Especially, we give some additional results for anti-invariant submersions from a cosymplectic manifold to a Riemannian manifold such that $\left(\operatorname{ker} F_{*}\right)^{\perp}=\phi\left(\operatorname{ker}\left(F_{*}\right)\right) \oplus\{\xi\}$.

\section{Riemannian submersions}

In this section we recall several notions and results which will be needed throughout the paper.

Let $\left(M, g_{M}\right)$ be an $m$-dimensional Riemannian manifold and let $\left(N, g_{N}\right)$ be an $n$-dimensional Riemannian manifold. A Riemannian submersion is a smooth map $F: M \rightarrow N$ which is onto and satisfying the following axioms:

$S 1$. $F$ has maximal rank.

$S 2$. The differential $F_{*}$ preserves the lengths of horizontal vectors.

The fundamental tensors of a submersion were defined by O'Neill ([20], [21]). They are $(1,2)$-tensors on $M$, given by the following formulas:

$$
\begin{aligned}
& \mathcal{T}(E, F)=\mathcal{T}_{E} F=\mathcal{H} \nabla_{\mathcal{V} E} \mathcal{V} F+\mathcal{V} \nabla_{\mathcal{V} E} \mathcal{H} F, \\
& \mathcal{A}(E, F)=\mathcal{A}_{E} F=\mathcal{V} \nabla_{\mathcal{H} E} \mathcal{H} F+\mathcal{H} \nabla_{\mathcal{H} E} \mathcal{V} F,
\end{aligned}
$$

for any vector fields $E$ and $F$ on $M$. Here $\nabla$ denotes the Levi-Civita connection of $\left(M, g_{M}\right)$. These tensors are called integrability tensors for the Riemannian submersions. Note that we denote the projection morphism on the distributions $\operatorname{ker} F_{*}$ and $\left(\operatorname{ker} F_{*}\right)^{\perp}$ by $\mathcal{V}$ and $\mathcal{H}$, respectively. The following lemmas are well known $([20],[21])$ : 
Lemma 1. For any $U, W$ vertical and $X, Y$ horizontal vector fields, the tensor fields $\mathcal{T}$ and $\mathcal{A}$ satisfy

$$
\text { i) } \begin{aligned}
\mathcal{T}_{U} W & =\mathcal{T}_{W} U, \\
\text { ii) } \mathcal{A}_{X} Y & =-\mathcal{A}_{Y} X=\frac{1}{2} \mathcal{V}[X, Y] .
\end{aligned}
$$

It is easy to see that $\mathcal{T}$ is vertical, $\mathcal{T}_{E}=\mathcal{T}_{\mathcal{V} E}$, and $\mathcal{A}$ is horizontal, $\mathcal{A}=\mathcal{A}_{\mathcal{H} E}$. For each $q \in N, F^{-1}(q)$ is an $(m-n)$-dimensional submanifold of $M$. The submanifolds $F^{-1}(q), q \in N$, are called fibers. A vector field on $M$ is called vertical if it is always tangent to fibers. A vector field on $M$ is called horizontal if it is always orthogonal to fibers. A vector field $X$ on $M$ is called basic if $X$ is horizontal and $F$-related to a vector field $X$ on $N$, i.e., $F_{*} X_{p}=X_{* F(p)}$ for all $p \in M$.

Lemma 2. Let $F:\left(M, g_{M}\right) \rightarrow\left(N, g_{N}\right)$ be a Riemannian submersion. If $X, Y$ are basic vector fields on $M$, then

i) $g_{M}(X, Y)=g_{N}\left(X_{*}, Y_{*}\right) \circ F$,

ii) $\mathcal{H}[X, Y]$ is basic and $F$-related to $\left[X_{*}, Y_{*}\right]$,

iii) $\mathcal{H}\left(\nabla_{X} Y\right)$ is a basic vector field corresponding to $\nabla_{X_{*}}^{*} Y_{*}$, where $\nabla^{*}$ is the connection on $N$.

iv) for any vertical vector field $V,[X, V]$ is vertical.

Moreover, if $X$ is basic and $U$ is vertical, then $\mathcal{H}\left(\nabla_{U} X\right)=\mathcal{H}\left(\nabla_{X} U\right)=\mathcal{A}_{X} U$. On the other hand, from (2.1) and (2.2) we have

$$
\begin{aligned}
& \nabla_{V} W=\mathcal{T}_{V} W+\hat{\nabla}_{V} W, \\
& \nabla_{V} X=\mathcal{H} \nabla_{V} X+\mathcal{T}_{V} X, \\
& \nabla_{X} V=\mathcal{A}_{X} V+\mathcal{V} \nabla_{X} V, \\
& \nabla_{X} Y=\mathcal{H} \nabla_{X} Y+\mathcal{A}_{X} Y,
\end{aligned}
$$

for $X, Y \in \Gamma\left(\left(\operatorname{ker} F_{*}\right)^{\perp}\right)$ and $V, W \in \Gamma\left(\operatorname{ker} F_{*}\right)$, where $\hat{\nabla}_{V} W=\mathcal{V} \nabla_{V} W$. On any fibre $F^{-1}(q), q \in N, \hat{\nabla}$ coincides with the Levi-Civita connection with respect to the metric induced by $g_{M}$. This induced metric on fibre $F^{-1}(q)$ is denoted by $\hat{g}$.

Notice that $\mathcal{T}$ acts on the fibres as the second fundamental form of the submersion and restricted to vertical vector fields and it can be easily seen that $\mathcal{T}=0$ is equivalent to the condition that the fibres are totally geodesic. A Riemannian submersion is called a Riemannian submersion with totally geodesic fibers if $\mathcal{T}$ vanishes identically. Let $U_{1}, \ldots, U_{m-n}$ be an orthonormal frame of $\Gamma\left(\operatorname{ker} F_{*}\right)$. Then the horizontal vector field $H=\frac{1}{m-n} \sum_{j=1}^{m-n} \mathcal{T}_{U_{j}} U_{j}$ is called the mean curvature vector field of the fiber. If $H=0$, then the Riemannian submersion is said to be minimal. A Riemannian submersion is called a Riemannian submersion with totally umbilical fibers if

$$
\mathcal{T}_{U} W=g_{M}(U, W) H
$$


for $U, W \in \Gamma\left(\operatorname{ker} F_{*}\right)$. For any $E \in \Gamma(T M), \mathcal{T}_{E}$ and $\mathcal{A}_{E}$ are skew-symmetric operators on $\left(\Gamma(T M), g_{M}\right)$ reversing the horizontal and the vertical distributions. By Lemma 1, horizontal distribution $\mathcal{H}$ is integrable if and only if $\mathcal{A}=0$. For any $D, E, G \in \Gamma(T M)$, one has

$$
g\left(\mathcal{T}_{D} E, G\right)+g\left(\mathcal{T}_{D} G, E\right)=0
$$

and

$$
g\left(\mathcal{A}_{D} E, G\right)+g\left(\mathcal{A}_{D} G, E\right)=0 .
$$

The tensor fields $\mathcal{A}, \mathcal{T}$ and their covariant derivatives play a fundamental role in expressing the Riemannian curvature $R$ of $(M, g)$. By (2.5) and (2.6), B. O'Neill [20] gave

$$
\begin{aligned}
R(S, W, V, U) & =g(R(S, W) V, U) \\
& =\hat{R}(S, W, V, U)+g\left(\mathcal{T}_{U} W, \mathcal{T}_{V} S\right)-g\left(\mathcal{T}_{V} W, \mathcal{T}_{U} S\right)
\end{aligned}
$$

where $\hat{R}$ is Riemannian curvature tensor of any fibre $\left(F^{-1}(q), \hat{g}_{q}\right)$. Precisely, if $\{U, V\}$ is an orthonormal basis of the vertical 2-plane, then the equation (2.12) implies that

$$
K(U \wedge V)=\hat{K}(U \wedge V)+\left\|\mathcal{T}_{U} V\right\|^{2}-g\left(\mathcal{T}_{U} U, \mathcal{T}_{V} V\right)
$$

where $K$ and $\hat{K}$ denote the sectional curvature of $M$ and fibre $F^{-1}(q)$, respectively. Moreover, the following formula was stated in [20]

$$
\begin{aligned}
R(Y, W, V, X)= & g\left(\left(\nabla_{X} \mathcal{T}\right)(V, W), Y\right)+g\left(\left(\nabla_{V} \mathcal{A}\right)(X, Y), W\right) \\
& -g\left(\mathcal{T}_{V} X, \mathcal{T}_{W} Y\right)+g\left(\mathcal{A}_{X} V, \mathcal{A}_{Y} W\right)
\end{aligned}
$$

for any $X, Y, Z \in \Gamma\left(\left(\operatorname{ker} F_{*}\right)^{\perp}\right), V, W \in \Gamma\left(\operatorname{ker} F_{*}\right)$.

We recall the notion of harmonic maps between Riemannian manifolds. Let $\left(M, g_{M}\right)$ and $\left(N, g_{N}\right)$ be Riemannian manifolds and suppose that $\varphi: M \rightarrow N$ is a smooth map between them. Then the differential $\varphi_{*}$ of $\varphi$ can be viewed as a section of the bundle $\operatorname{Hom}\left(T M, \varphi^{-1} T N\right) \rightarrow M$, where $\varphi^{-1} T N$ is the pullback bundle which has fibres $\left(\varphi^{-1} T N\right)_{p}=T_{\varphi(p)} N, p \in M$. Hom $\left(T M, \varphi^{-1} T N\right)$ has a connection $\nabla$ induced from the Levi-Civita connection $\nabla^{M}$ and the pullback connection. Then the second fundamental form of $\varphi$ is given by

$$
\left(\nabla \varphi_{*}\right)(X, Y)=\nabla_{X}^{\varphi} \varphi_{*}(Y)-\varphi_{*}\left(\nabla_{X}^{M} Y\right)
$$

for $X, Y \in \Gamma(T M)$, where $\nabla^{\varphi}$ is the pullback connection. It is known that the second fundamental form is symmetric. If $\varphi$ is a Riemannian submersion, it can be easily proved that

$$
\left(\nabla \varphi_{*}\right)(X, Y)=0
$$

for $X, Y \in \Gamma\left(\left(\operatorname{ker} F_{*}\right)^{\perp}\right)$. A smooth map $\varphi:\left(M, g_{M}\right) \rightarrow\left(N, g_{N}\right)$ is said to be harmonic if $\operatorname{trace}\left(\nabla \varphi_{*}\right)=0$. On the other hand, the tension field of $\varphi$ is the 
section $\tau(\varphi)$ of $\Gamma\left(\varphi^{-1} T N\right)$ defined by

$$
\tau(\varphi)=\operatorname{div} \varphi_{*}=\sum_{i=1}^{m}\left(\nabla \varphi_{*}\right)\left(e_{i}, e_{i}\right),
$$

where $\left\{e_{1}, \ldots, e_{m}\right\}$ is the orthonormal frame on $M$. Then it follows that $\varphi$ is harmonic if and only if $\tau(\varphi)=0$; for details, see [1].

\section{Cosymplectic manifolds}

A $(2 m+1)$-dimensional $C^{\infty}$-manifold $M$ is said to have an almost contact structure if there exist a tensor field $\phi$ of type $(1,1)$ on $M$, a vector field $\xi$ and 1-form $\eta$ satisfying

$$
\phi^{2}=-I+\eta \otimes \xi, \quad \phi \xi=0, \eta \circ \phi=0, \quad \eta(\xi)=1 .
$$

There always exists a Riemannian metric $g$ on an almost contact manifold $M$ satisfying the following conditions

$$
g(\phi X, \phi Y)=g(X, Y)-\eta(X) \eta(Y), \eta(X)=g(X, \xi),
$$

where $X, Y$ are vector fields on $M$.

An almost contact structure $(\phi, \xi, \eta)$ is said to be normal if the almost complex structure $J$ on the product manifold $M \times R$ is given by

$$
J\left(X, f \frac{d}{d t}\right)=\left(\phi X-f \xi, \eta(X) \frac{d}{d t}\right),
$$

where $f$ is the $C^{\infty}$-function on $M \times R$ has no torsion, i.e., $J$ is integrable. The condition for normality in terms of $\phi, \xi$ and $\eta$ is $[\phi, \phi]+2 d \eta \otimes \xi=0$ on $M$, where $[\phi, \phi]$ is the Nijenhuis tensor of $\phi$. Finally, the fundamental 2-form $\Phi$ is defined by $\Phi(X, Y)=g(X, \phi Y)$.

An almost contact metric structure $(\phi, \xi, \eta, g)$ is said to be cosymplectic, if it is normal and both $\Phi$ and $\eta$ are closed ([2], [16]), and the structure equation of a cosymplectic manifold is given by

$$
\left(\nabla_{E} \phi\right) G=0
$$

for any $E, G$ tangent to $M$, where $\nabla$ denotes the Riemannian connection of the metric $g$ on $M$. Moreover, for a cosymplectic manifold, we know that

$$
\nabla_{E} \xi=0 \text {. }
$$

The $\phi$-sectional curvature of a cosymplectic manifold $M$ is defined for any unit vector $E$ on $M$ orthogonal to $\xi$ by the formula

$$
H(E)=g(R(E, \phi E) \phi E, E) .
$$

If a cosymplectic manifold $M$ has $\phi$-sectional curvature $c$, then $M$ is called a cosymplectic space form and denoted by $M(c)$, [31]. So the curvature tensor $R$ of a cosymplectic space form $M(c)$ is given by

$$
R(X, Y) Z=\frac{c}{4}(g(Y, Z) X-g(X, Z) Y+\eta(X) \eta(Z) Y-\eta(Y) \eta(Z) X
$$




$$
\begin{aligned}
& +g(X, Z) \eta(Y) \xi) \\
& -g(Y, Z) \eta(X) \xi+g(\phi Y, Z) \phi X-g(\phi X, Z) \phi Y-2 g(\phi X, Y) \phi Z),
\end{aligned}
$$

in [31] for any tangent vector fields $X, Y, Z$ to $M(c)$.

The canonical example of cosymplectic manifold is given by the product $B^{2 n} \times \mathbb{R}$ of the Kähler manifold $B^{2 n}(J, g)$ with the real line $\mathbb{R}$. Now we will give some well known examples which are cosymplectic manifolds on $\mathbb{R}^{2 n+1}$.

Example 1 ([19]). We consider $\mathbb{R}^{2 n+1}$ with Cartesian coordinates $\left(x_{i}, y_{i}, z\right)$ $(i=1, \ldots, n)$ and its usual contact form

$$
\eta=d z
$$

The characteristic vector field $\xi$ is given by $\frac{\partial}{\partial z}$ and its Riemannian metric $g$ and tensor field $\phi$ are given by

$$
g=\sum_{i=1}^{n}\left(\left(d x_{i}\right)^{2}+\left(d y_{i}\right)^{2}\right)+(d z)^{2}, \quad \phi=\left(\begin{array}{ccc}
0 & \delta_{i j} & 0 \\
-\delta_{i j} & 0 & 0 \\
0 & 0 & 0
\end{array}\right), i=1, \ldots, n
$$

This gives a cosymplectic structure on $\mathbb{R}^{2 n+1}$. The vector fields $E_{i}=\frac{\partial}{\partial y_{i}}$, $E_{n+i}=\frac{\partial}{\partial x_{i}}, \xi$ form a $\phi$-basis for the cosymplectic structure. On the other hand, it can be shown that $\mathbb{R}^{2 n+1}(\phi, \xi, \eta, g)$ is a cosymplectic manifold.

Example 2 ([14]). We denote the Cartesian coordinates in $\mathbb{R}^{5}$ by $\left(x_{1}, x_{2}, x_{3}\right.$, $\left.x_{4}, x_{5}\right)$ and its Riemannian metric $g$ by

$$
g=\left(\begin{array}{ccccc}
1+\tau^{2} & 0 & \tau^{2} & 0 & -\tau \\
0 & 1 & 0 & 0 & 0 \\
\tau^{2} & 0 & 1+\tau^{2} & 0 & -\tau \\
0 & 0 & 0 & 1 & 0 \\
-\tau & 0 & -\tau & 0 & 1
\end{array}\right)
$$

where $\tau=\sin \left(x_{1}+x_{3}\right)$. We define an almost contact structure $(\phi, \xi, \eta)$ on $\mathbb{R}^{5}$ by

$$
\phi=\left(\begin{array}{ccccc}
0 & -1 & 0 & 0 & 0 \\
1 & 0 & 0 & 0 & 0 \\
0 & 0 & 0 & -1 & 0 \\
0 & 0 & 1 & 0 & 0 \\
0 & -\tau & 0 & -\tau & 0
\end{array}\right), \eta=-\tau d x_{1}-\tau d x_{3}+d x_{5}, \quad \xi=\frac{\partial}{\partial x_{5}}
$$

The fundamental 2-form $\Phi$ has the form

$$
\Phi=-\left(d x_{1} \wedge d x_{2}+d x_{3} \wedge d x_{4}\right) .
$$

This gives a cosymplectic structure on $\mathbb{R}^{5}$. If we take vector fields $E_{1}=\frac{\partial}{\partial x_{1}}+$ $\tau \frac{\partial}{\partial x_{5}}, E_{2}=\frac{\partial}{\partial x_{3}}+\tau \frac{\partial}{\partial x_{5}}, \phi E_{1}=E_{3}=\frac{\partial}{\partial x_{2}}, \phi E_{2}=E_{4}=\frac{\partial}{\partial x_{4}}$ and $E_{5}=\frac{\partial}{\partial x_{5}}$, then these vector fields form a frame field in $\mathbb{R}^{5}$. 


\section{Slant Riemannian submersions}

Definition 1. Let $M\left(\phi, \xi, \eta, g_{M}\right)$ be a cosymplectic manifold and $\left(N, g_{N}\right)$ be a Riemannian manifold. A Riemannian submersion $F: M\left(\phi, \xi, \eta, g_{M}\right) \rightarrow\left(N, g_{N}\right)$ is said to be slant if for any non zero vector $X \in \Gamma\left(\operatorname{ker} F_{*}\right)-\{\xi\}$, the angle $\theta(X)$ between $\phi X$ and the space ker $F_{*}$ is a constant (which is independent of the choice of $p \in M$ and of $\left.X \in \Gamma\left(\operatorname{ker} F_{*}\right)-\{\xi\}\right)$. The angle $\theta$ is called the slant angle of the slant submersion. Invariant and anti-invariant submersions are slant submersions with $\theta=0$ and $\theta=\pi / 2$, respectively. A slant submersion which is not invariant nor anti-invariant is called proper submersion.

Now we will give some examples.

Example 3. $\mathbb{R}^{5}$ has got a cosymplectic structure as in Example 1. Let $F$ : $\mathbb{R}^{5} \rightarrow \mathbb{R}^{2}$ be a map defined by $F\left(x_{1}, x_{2}, y_{1}, y_{2}, z\right)=\left(\frac{1}{\sqrt{2}}\left(x_{1}-x_{2}\right), y_{2}\right)$. Then, by direct calculations we have

$$
\operatorname{ker} F_{*}=\operatorname{span}\left\{V_{1}=E_{1}, V_{2}=\frac{1}{\sqrt{2}}\left(E_{3}+E_{4}\right), V_{3}=\xi=E_{5}\right\}
$$

and

$$
\left(\operatorname{ker} F_{*}\right)^{\perp}=\operatorname{span}\left\{H_{1}=\frac{1}{\sqrt{2}}\left(E_{3}-E_{4}\right), H_{2}=E_{2}\right\} .
$$

Then it is easy to see that $F$ is a Riemannian submersion. Moreover, $\phi V_{1}=E_{3}$ and $\phi V_{2}=-\frac{1}{\sqrt{2}}\left(E_{1}+E_{2}\right)$ imply that $\left|g\left(\phi V_{1}, V_{2}\right)\right|=\frac{1}{\sqrt{2}}$. So $F$ is a slant submersion with slant angle $\theta=\frac{\pi}{4}$ and $\xi$ is a vertical vector field.

Example 4. $\mathbb{R}^{5}$ has got a cosymplectic structure as in Example 2. Let $F$ : $\mathbb{R}^{5} \rightarrow \mathbb{R}^{2}$ be a map defined by $F\left(x_{1}, x_{2}, y_{1}, y_{2}, z\right)=\left(\frac{1}{\sqrt{2}}\left(x_{1}-y_{1}\right), \frac{1}{\sqrt{2}}\left(x_{2}-y_{2}\right)\right)$. Then, a simple calculation gives

$$
\text { ker } F_{*}=\operatorname{span}\left\{V_{1}=\frac{1}{\sqrt{2}}\left(E_{3}+E_{4}\right), V_{2}=\frac{1}{\sqrt{2}}\left(E_{1}+E_{2}\right), V_{3}=\xi=E_{5}\right\}
$$

and

$$
\left(\operatorname{ker} F_{*}\right)^{\perp}=\operatorname{span}\left\{H_{1}=\frac{1}{\sqrt{2}}\left(E_{3}-E_{4}\right), H_{2}=\frac{1}{\sqrt{2}}\left(E_{1}-E_{2}\right)\right\} .
$$

Then it is easy to see that $F$ is a Riemannian submersion. Moreover, $\phi V_{1}=$ $-\frac{1}{\sqrt{2}}\left(E_{1}+E_{2}\right)$ and $\phi V_{2}=\frac{1}{\sqrt{2}}\left(E_{3}+E_{4}\right)$ imply that $\left|g\left(\phi V_{1}, V_{2}\right)\right|=1$. So $F$ is a slant submersion with slant angle $\theta=0$.

Example 5. $\mathbb{R}^{5}$ has got a cosymplectic structure as in Example 1. Let $F$ : $\mathbb{R}^{5} \rightarrow \mathbb{R}^{3}$ be a map defined by $F\left(x_{1}, x_{2}, y_{1}, y_{2}, z\right)=\left(\frac{1}{\sqrt{2}}\left(x_{1}-x_{2}\right), y_{2}, z\right)$. Then, by direct calculations we obtain

$$
\operatorname{ker} F_{*}=\operatorname{span}\left\{V_{1}=E_{1}, V_{2}=\frac{1}{\sqrt{2}}\left(E_{3}+E_{4}\right)\right\}
$$

and

$$
\left(\operatorname{ker} F_{*}\right)^{\perp}=\operatorname{span}\left\{H_{1}=\frac{1}{\sqrt{2}}\left(E_{3}-E_{4}\right), H_{2}=E_{2}, H_{3}=\xi\right\} .
$$


Then it is easy to see that $F$ is a Riemannian submersion. Moreover, $\phi V_{1}=E_{3}$ and $\phi V_{2}=-\frac{1}{\sqrt{2}}\left(E_{1}+E_{2}\right)$ imply that $\left|g\left(\phi V_{1}, V_{2}\right)\right|=\frac{1}{\sqrt{2}}$. So $F$ is a slant submersion with slant angle $\theta=\frac{\pi}{4}$ and $\xi$ is a horizontal vector field.

\subsection{Slant Riemannian submersions admitting vertical structure vec- tor field}

In this subsection, we will investigate the properties of slant Riemannian submersions from a cosymplectic manifold onto a Riemannian manifold such that characteristic vector field $\xi$ is a vertical vector field.

Now, let $F$ be a slant Riemannian submersion from a cosymplectic manifold $M\left(\phi, \xi, \eta, g_{M}\right)$ onto a Riemannian manifold $\left(N, g_{N}\right)$. Then for any $U, V \in$ $\Gamma\left(\operatorname{ker} F_{*}\right)$, we put

$$
\phi U=\psi U+\omega U,
$$

where $\psi U$ and $\omega U$ are vertical and horizontal components of $\phi U$, respectively. Similarly, for any $X \in \Gamma\left(\operatorname{ker} F_{*}\right)^{\perp}$, we have

$$
\phi X=\mathcal{B} X+\mathcal{C} X
$$

where $\mathcal{B} X$ (resp. $\mathcal{C} X$ ) is vertical part (resp. horizontal part) of $\phi X$.

From (3.2), (4.1) and (4.2) we obtain

$$
g_{M}(\psi U, V)=-g_{M}(U, \psi V)
$$

and

$$
g_{M}(\omega U, Y)=-g_{M}(U, \mathcal{B} Y)
$$

for any $U, V \in \Gamma\left(\operatorname{ker} F_{*}\right)$ and $Y \in \Gamma\left(\left(\operatorname{ker} F_{*}\right)^{\perp}\right)$.

Using (2.5), (2.7) and (3.4) we obtain

$$
\mathcal{T}_{U} \xi=0, \mathcal{A}_{X} \xi=0
$$

for any $U \in \Gamma\left(\operatorname{ker} F_{*}\right), X \in \Gamma\left(\left(\operatorname{ker} F_{*}\right)^{\perp}\right)$.

From (3.1), (4.1) and (4.2) we can easily obtain following lemma.

Lemma 3. Let $F$ be a slant Riemannian submersion from a cosymplectic manifold $M\left(\phi, \xi, \eta, g_{M}\right)$ to a Riemannian manifold $\left(N, g_{N}\right)$. Then we have

$$
\begin{aligned}
-X & =\omega \mathcal{B} X+\mathcal{C}^{2} X, \\
0 & =\psi \mathcal{B} X+\mathcal{B C} X, \\
\phi^{2} U & =\psi^{2} U+\mathcal{B} \omega U, \\
0 & =\omega \psi U+\mathcal{C} \omega U, \\
g_{M}(\mathcal{C} X, \phi U) & =-g_{M}(\mathcal{B} X, \psi U)
\end{aligned}
$$

for any $X \in \Gamma\left(\left(\operatorname{ker} F_{*}\right)^{\perp}\right)$ and $V \in \Gamma\left(\left(\operatorname{ker} F_{*}\right)\right)$.

Proposition 1 ([15]). Let $F$ be a Riemannian submersion from an almost contact manifold onto a Riemannian manifold. If $\operatorname{dim}\left(\operatorname{ker} F_{*}\right)=2$ and $\xi$ is a vertical vector field, then fibers are anti-invariant. 
Theorem 1. Let $M\left(\phi, \xi, \eta, g_{M}\right)$ be a cosymplectic manifold of dimension $2 m+1$ and $\left(N, g_{N}\right)$ is a Riemannian manifold of dimension $n$. Let $F: M\left(\phi, \xi, \eta, g_{M}\right)$ $\rightarrow\left(N, g_{N}\right)$ be a slant Riemannian submersion. Then the fibers are not proper totally umbilical.

Proof. If the fibers are proper totally umbilical, then we have

$$
\mathcal{T}_{U} V=g_{M}(U, V) H
$$

for any vertical vector fields $U, V$ where $H$ is the mean curvature vector field of any fibre. Since $\mathcal{T}_{\xi} \xi=0$, we have $H=0$, which shows that fibres are minimal. Hence the fibers are totally geodesic. This completes the proof of the theorem.

By (2.5), (2.6), (4.1) and (4.2), we have

$$
\begin{aligned}
& \left(\nabla_{U} \omega\right) V=\mathcal{C} T_{U} V-\mathcal{T}_{U} \psi V \\
& \left(\nabla_{U} \psi\right) V=\mathcal{B} \mathcal{T}_{U} V-\mathcal{T}_{U} \omega V
\end{aligned}
$$

where

$$
\begin{aligned}
& \left(\nabla_{U} \omega\right) V=\mathcal{H} \nabla_{U} \omega V-\omega \hat{\nabla}_{U} V \\
& \left(\nabla_{U} \psi\right) V=\hat{\nabla}_{U} \psi V-\psi \hat{\nabla}_{U} V
\end{aligned}
$$

for $U, V \in \Gamma\left(\operatorname{ker} F_{*}\right)$.

We will give a characterization theorem for slant submersions of a cosymplectic manifold. Since the proof of the following theorem is quite similar to Theorem 3 of [15], so we don't give the proof of it.

Theorem 2. Let $F$ be a Riemannian submersion from a cosymplectic manifold $M\left(\phi, \xi, \eta, g_{M}\right)$ onto a Riemannian manifold $\left(N, g_{N}\right)$ such that $\xi \in \Gamma\left(\operatorname{ker} F_{*}\right)$. Then, $F$ is a slant Riemannian submersion if and only if there exists a constant $\lambda \in[0,1]$ such that

$$
\psi^{2}=-\lambda(I-\eta \otimes \xi)
$$

Furthermore, in such a case, if $\theta$ is the slant angle of $F$, it satisfies that $\lambda=$ $\cos ^{2} \theta$.

By using (3.2), (4.1), (4.3) and (4.10), we have the following lemma.

Lemma 4. Let $F$ be a slant Riemannian submersion from a cosymplectic manifold $M\left(\phi, \xi, \eta, g_{M}\right)$ onto a Riemannian manifold $\left(N, g_{N}\right)$ with slant angle $\theta$. Then the following relations are valid

$$
\begin{aligned}
& g_{M}(\psi U, \psi V)=\cos ^{2} \theta\left(g_{M}(U, V)-\eta(U) \eta(V)\right), \\
& g_{M}(\omega U, \omega V)=\sin ^{2} \theta\left(g_{M}(U, V)-\eta(U) \eta(V)\right),
\end{aligned}
$$

for any $U, V \in \Gamma\left(\operatorname{ker} F_{*}\right)$. 
We denote the complementary orthogonal distribution to $\omega\left(\right.$ ker $\left.F_{*}\right)$ in $\left(\text { ker } F_{*}\right)^{\perp}$ by $\mu$. Then we have

$$
\left(\operatorname{ker} F_{*}\right)^{\perp}=\omega\left(\operatorname{ker} F_{*}\right) \oplus \mu .
$$

Lemma 5. Let $F$ be a proper slant Riemannian submersion from a cosymplectic manifold $M\left(\phi, \xi, \eta, g_{M}\right)$ onto a Riemannian manifold $\left(N, g_{N}\right)$. Then $\mu$ is an invariant distribution of $\left(\operatorname{ker} F_{*}\right)^{\perp}$, under the endomorphism $\phi$.

Proof. For $X \in \Gamma(\mu)$ and $V \in \Gamma\left(\operatorname{ker} F_{*}\right)$, from (3.2) and (4.1), we obtain

$$
\begin{aligned}
g_{M}(\phi X, \omega V) & =g_{M}(\phi X, \phi V)-g_{M}(\phi X, \psi V) \\
& =g_{M}(X, V)-\eta(X) \eta(V)-g_{M}(\phi X, \psi V) \\
& =g_{M}(X, \phi \psi V)=0 .
\end{aligned}
$$

In a similar way, we have $g_{M}(\phi X, U)=-g_{M}(X, \phi U)=0$ due to $\phi U \in$ $\Gamma\left(\left(\operatorname{ker} F_{*}\right) \oplus \omega\left(\operatorname{ker} F_{*}\right)\right)$ for $X \in \Gamma(\mu)$ and $U \in \Gamma\left(\operatorname{ker} F_{*}\right)$. Thus the proof of the lemma is completed.

By help (4.12), we can give the following result:

Corollary 1. Let $F$ be a proper slant Riemannian submersion from a cosymplectic manifold $M^{2 m+1}\left(\phi, \xi, \eta, g_{M}\right)$ onto a Riemannian manifold $\left(N^{n}, g_{N}\right)$. Let $\left\{e_{1}, e_{2}, \ldots, e_{2 m-n}, \xi\right\}$ be a local orthonormal frame of (ker $\left.F_{*}\right)$. Then

$$
\left\{\csc \theta \omega e_{1}, \csc \theta \omega e_{2}, \ldots, \csc \theta \omega e_{2 m-n}\right\}
$$

is a local orthonormal frame of $\omega\left(\operatorname{ker} F_{*}\right)$.

By using (4.13) and Corollary 1, one can easily prove the following proposition.

Proposition 2. Let $F$ be a proper slant Riemannian submersion from a cosymplectic manifold $M^{2 m+1}\left(\phi, \xi, \eta, g_{M}\right)$ onto a Riemannian manifold $\left(N^{n}, g_{N}\right)$. Then $\operatorname{dim}(\mu)=2(n-m)$. If $\mu=\{0\}$, then $n=m$.

Lemma 6. Let $F$ be a proper slant Riemannian submersion from a cosymplectic manifold $M^{2 m+1}\left(\phi, \xi, \eta, g_{M}\right)$ onto a Riemannian manifold $\left(N^{2 n}, g_{N}\right)$ such that $\xi$ is vertical. Then there exists a local orthonormal frame

$$
\left\{e_{1}, \sec \theta \psi e_{1}, e_{2}, \sec \theta \psi e_{2}, \ldots, e_{m-n}, \sec \theta \psi e_{m-n}, \xi\right\}
$$

of $\left(\operatorname{ker} F_{*}\right)$.

Proof. Let $e_{1}$ be a unit vector field of (ker $\left.F_{*}\right)$ such that it is perpendicular to $\xi$. We put $e_{2}^{*}=\sec \theta \psi e_{1}$. By (4.11), we have $e_{2}^{*} \perp\left\{e_{1}, \xi\right\}$ and $g_{M}\left(\sec \theta \psi e_{1}, \sec \theta \psi e_{1}\right)$ $=1$. Now we choose a unit vector field $e_{2}$ orthogonal to $e_{1}, e_{2}^{*}=\sec \theta \psi e_{1}$ and $\xi$. Then $\sec \theta \psi e_{2}$ is also a vector field orthogonal to $e_{1}, \sec \theta \psi e_{1}, \xi$ and $e_{2}$, and also $g_{M}\left(\sec \theta \psi e_{2}, \sec \theta \psi e_{2}\right)=1$. Proceeding in this way, we obtain a local orthonormal frame $\left\{e_{i}, e_{i}^{*}=\sec \theta \psi e_{i}, \xi: i=1, \ldots, m-n\right\}$ of $\left(\operatorname{ker} F_{*}\right)$. 
Lemma 7. Let $F$ be a slant Riemannian submersion from a cosymplectic manifold $M\left(\phi, \xi, \eta, g_{M}\right)$ onto a Riemannian manifold $\left(N, g_{N}\right)$. If $\omega$ is parallel, then we have

$$
\mathcal{T}_{\psi U} \psi U=-\cos ^{2} \theta \mathcal{T}_{U} U
$$

Proof. If $\omega$ is parallel, from (4.6), we obtain $\mathcal{C} T_{U} V=\mathcal{T}_{U} \psi V$ for $U, V \in$ $\Gamma\left(\operatorname{ker} F_{*}\right)$. After putting $V$ instead of $U$ and using (2.3), we obtain

$$
\mathcal{T}_{U} \psi V=\mathcal{T}_{V} \psi U .
$$

Substituting $V$ by $\psi U$ in the above equation and using Theorem 2, we get the required formula.

We give a sufficient condition for a slant Riemannian submersion to be harmonic as an analogue of a slant Riemannian submersion from an almost Hermitian manifold onto a Riemannian manifold in [25].

Theorem 3. Let $F$ be a slant Riemannian submersion from a cosymplectic manifold $M\left(\phi, \xi, \eta, g_{M}\right)$ onto a Riemannian manifold $\left(N, g_{N}\right)$. If $\omega$ is parallel, then $F$ is a harmonic map.

Proof. The proof is similar to the proof of Theorem 4 in [15] and [25].

Now putting $Q=\psi^{2}$, we define $\nabla Q$ by

$$
\left(\nabla_{U} Q\right) V=\mathcal{V} \nabla_{U} Q V-Q \hat{\nabla}_{U} V
$$

for any $U, V \in \Gamma\left(\operatorname{ker} F_{*}\right)$.

Theorem 4. Let $F$ be a slant Riemannian submersion from a cosymplectic manifold $M\left(\phi, \xi, \eta, g_{M}\right)$ onto a Riemannian manifold $\left(N, g_{N}\right)$. Then $\nabla Q=0$.

Proof. From (4.10),

$$
Q \hat{\nabla}_{U} V=-\cos ^{2} \theta\left(\hat{\nabla}_{U} V-\eta\left(\hat{\nabla}_{U} V\right) \xi\right)
$$

for each $U, V \in \Gamma\left(\operatorname{ker} F_{*}\right)$, where $\theta$ is the slant angle. On the other hand, the following equation is valid,

$$
\mathcal{V}\left(\nabla_{U} Q V\right)=-\cos ^{2} \theta\left(\hat{\nabla}_{U} V-\eta\left(\hat{\nabla}_{U} V\right) \xi .\right.
$$

Combining (4.16) and (4.17), we obtain $\left(\nabla_{U} Q\right) V=0$. This completes the proof.

We now investigate the geometry of leaves of $\left(\operatorname{ker} F_{*}\right)^{\perp}$ and ker $F_{*}$.

Theorem 5. Let $F$ be a proper slant Riemannian submersion from a cosymplectic manifold $M\left(\phi, \xi, \eta, g_{M}\right)$ onto a Riemannian manifold $\left(N, g_{N}\right)$. Then the distribution $\left(\operatorname{ker} F_{*}\right)^{\perp}$ defines a totally geodesic foliation on $M$ if and only if

$$
g_{M}\left(\mathcal{H} \nabla_{X} Y, \omega \psi U\right)=g_{M}\left(\mathcal{A}_{X} \mathcal{B} Y, \omega U\right)+g_{M}\left(\mathcal{H} \nabla_{X} \mathcal{C} Y, \omega U\right)
$$

for any $X, Y \in \Gamma\left(\left(\operatorname{ker} F_{*}\right)^{\perp}\right)$ and $U \in \Gamma\left(\operatorname{ker} F_{*}\right)$. 
Proof. From (3.3) and (4.1), we have

$$
\begin{aligned}
g_{M}\left(\nabla_{X} Y, U\right) & =-g_{M}\left(\phi \nabla_{X} \phi Y, U\right)+g_{M}\left(\nabla_{X} Y, \xi\right) \eta(U) \\
& =g_{M}\left(\nabla_{X} \phi Y, \phi U\right)+g_{M}\left(\nabla_{X} Y, \xi\right) \eta(U) \\
& =g_{M}\left(\phi \nabla_{X} Y, \psi U\right)+g_{M}\left(\nabla_{X} \phi Y, \omega U\right)+g_{M}\left(\nabla_{X} Y, \xi\right) \eta(U)
\end{aligned}
$$

for any $X, Y \in \Gamma\left(\left(\operatorname{ker} F_{*}\right)^{\perp}\right)$ and $U \in \Gamma\left(\operatorname{ker} F_{*}\right)$.

Using (3.3) and (4.1) in (4.18), we obtain

$$
\begin{aligned}
g_{M}\left(\nabla_{X} Y, U\right)= & -g_{M}\left(\nabla_{X} Y, \psi^{2} U\right)-g_{M}\left(\nabla_{X} Y, \omega \psi U\right) \\
& +g_{M}\left(\nabla_{X} Y, \xi\right) \eta(U)+g_{M}\left(\nabla_{X} \phi Y, \omega U\right) .
\end{aligned}
$$

By (4.2) and (4.10) we have

$$
\begin{aligned}
g_{M}\left(\nabla_{X} Y, U\right)= & \cos ^{2} \theta g_{M}\left(\nabla_{X} Y, U\right)-\cos ^{2} \theta \eta(U) \eta\left(\nabla_{X} Y\right) \\
& -g_{M}\left(\nabla_{X} Y, \omega \psi U\right)+g_{M}\left(\nabla_{X} Y, \xi\right) \eta(U) \\
& +g_{M}\left(\nabla_{X} \mathcal{B} Y, \omega U\right)+g_{M}\left(\nabla_{X} \mathcal{C} Y, \omega U\right) .
\end{aligned}
$$

Using (2.7), (2.8) and (3.4) in the last equation, we obtain

$$
g_{M}\left(\mathcal{H} \nabla_{X} Y, \omega \psi U\right)=g_{M}\left(\mathcal{A}_{X} \mathcal{B} Y, \omega U\right)+g_{M}\left(\mathcal{H} \nabla_{X} \mathcal{C} Y, \omega U\right)
$$

which proves the theorem.

Theorem 6. Let $F$ be a proper slant Riemannian submersion from a cosymplectic manifold $M\left(\phi, \xi, \eta, g_{M}\right)$ onto a Riemannian manifold $\left(N, g_{N}\right)$. Then the distribution $\left(\operatorname{ker} F_{*}\right)$ defines a totally geodesic foliation on $M$ if and only if

$$
g_{M}\left(\mathcal{H} \nabla_{U} \omega \psi V, X\right)=g_{M}\left(\mathcal{T}_{U} \omega V, \mathcal{B} X\right)+g_{M}\left(\mathcal{H} \nabla_{U} \omega V, \mathcal{C} X\right)
$$

for any $U, V \in \Gamma\left(\operatorname{ker} F_{*}\right)$ and $X \in \Gamma\left(\left(\operatorname{ker} F_{*}\right)^{\perp}\right)$.

Proof. From (3.3) and (4.1), we have

$$
\begin{aligned}
g_{M}\left(\nabla_{U} V, X\right) & =-g_{M}\left(\phi \nabla_{U} \phi V, X\right)+g_{M}\left(\nabla_{U} V, \xi\right) \eta(X) \\
& =-g_{M}\left(\phi \nabla_{U} \psi V, X\right)-g_{M}\left(\phi \nabla_{U} \omega V, X\right) \\
& =-g_{M}\left(\phi \nabla_{U} \psi V, X\right)+g_{M}\left(\nabla_{U} \omega V, \phi X\right)
\end{aligned}
$$

for any $U, V \in \Gamma\left(\operatorname{ker} F_{*}\right)$ and $X \in \Gamma\left(\left(\operatorname{ker} F_{*}\right)^{\perp}\right)$.

Using (3.3), (4.1) and (4.2) in (4.21), we obtain

$$
\begin{aligned}
g_{M}\left(\nabla_{U} V, X\right)= & -g_{M}\left(\nabla_{U} \psi^{2} V, X\right)-g_{M}\left(\nabla_{U} \omega \psi V, X\right) \\
& +g_{M}\left(\nabla_{U} \omega V, \mathcal{B} X\right)+g_{M}\left(\nabla_{U} \omega V, \mathcal{C} X\right) .
\end{aligned}
$$

By using (4.10), (2.7), (2.8) and (3.4) in the last equation, we obtain the requested relation.

From Theorem 5 and Theorem 6 we have the following corollary. 
Corollary 2. Let $F$ be a proper slant Riemannian submersion from a cosymplectic manifold $M\left(\phi, \xi, \eta, g_{M}\right)$ onto a Riemannian manifold $\left(N, g_{N}\right)$. Then $M$ is a locally product Riemannian manifold if and only if

$$
g_{M}\left(\mathcal{H} \nabla_{U} \omega \psi V, X\right)=g_{M}\left(\mathcal{T}_{U} \omega V, \mathcal{B} X\right)+g_{M}\left(\mathcal{H} \nabla_{U} \omega V, \mathcal{C} X\right)
$$

and

$$
g_{M}\left(\mathcal{H} \nabla_{X} Y, \omega \psi U\right)=g_{M}\left(\mathcal{A}_{X} \mathcal{B} Y, \omega U\right)+g_{M}\left(\mathcal{H} \nabla_{X} \mathcal{C} Y, \omega U\right)
$$

for any $U, V \in \Gamma\left(\operatorname{ker} F_{*}\right)$ and $X \in \Gamma\left(\left(\operatorname{ker} F_{*}\right)^{\perp}\right)$.

For a slant Riemannian submersion we have the following theorem.

Theorem 7. Let $F$ be a proper slant Riemannian submersion from a cosymplectic manifold $M\left(\phi, \xi, \eta, g_{M}\right)$ onto a Riemannian manifold $\left(N, g_{N}\right)$. Then $F$ is a totally geodesic map if and only if

$$
g_{M}\left(\mathcal{H} \nabla_{U} \omega \psi V, X\right)=g_{M}\left(\mathcal{T}_{U} \omega V, \mathcal{B} X\right)+g_{M}\left(\mathcal{H} \nabla_{U} \omega V, \mathcal{C} X\right)
$$

and

$$
g_{M}\left(\mathcal{H} \nabla_{Y} \omega \psi U, X\right)=g_{M}\left(\mathcal{A}_{Y} \omega U, \mathcal{B} X\right)+g_{M}\left(\mathcal{H} \nabla_{Y} \omega U, \mathcal{C} X\right)
$$

for $U, V \in \Gamma\left(\operatorname{ker} F_{*}\right)$ and $X, Y \in \Gamma\left(\left(\operatorname{ker} F_{*}\right)^{\perp}\right)$.

Proof. First of all, we recall that a Riemannian submersion satisfies

$$
\left(\nabla F_{*}\right)(X, Y)=0
$$

for $X, Y \in \Gamma\left(\left(\operatorname{ker} F_{*}\right)^{\perp}\right)$. We will prove that $\left(\nabla F_{*}\right)(U, V)=0$ and $\left(\nabla F_{*}\right)(X, U)$ $=0$ for $U, V \in \Gamma\left(\operatorname{ker} F_{*}\right)$ and $X \in \Gamma\left(\left(\operatorname{ker} F_{*}\right)^{\perp}\right)$. Since $F$ is a Riemannian submersion, from (3.1) and (3.3) we get

$$
\nabla_{U} V=-\phi \nabla_{U} \phi V+\eta\left(\nabla_{U} V\right) \xi .
$$

From (4.1) and (4.23), we obtain

$$
g_{N}\left(\left(\nabla F_{*}\right)(U, V), F_{*} X\right)=g_{M}\left(\nabla_{U} \phi \psi V, X\right)-g_{M}\left(\nabla_{U} \omega V, \phi X\right) .
$$

Using (4.1) and (4.2) once more, we get

$$
\begin{aligned}
g_{N}\left(\left(\nabla F_{*}\right)(U, V), F_{*} X\right)= & g_{M}\left(\nabla_{U} \psi^{2} V, X\right)+g_{M}\left(\nabla_{U} \omega \psi V, X\right) \\
& -g_{M}\left(\nabla_{U} \omega V, \mathcal{B} X\right)-g_{M}\left(\nabla_{U} \omega V, \mathcal{C} X\right) .
\end{aligned}
$$

Theorem 2, (2.5) and (2.6) imply that

$$
\begin{aligned}
g_{N}\left(\left(\nabla F_{*}\right)(U, V), F_{*} X\right)= & -\cos ^{2} \theta g_{M}\left(\nabla_{U} V, X\right)+g_{M}\left(\nabla_{U} \omega \psi V, X\right) \\
& -g_{M}\left(\mathcal{T}_{U} \omega V, \mathcal{B} X\right)-g_{M}\left(\mathcal{H} \nabla_{U} \omega V, \mathcal{C} X\right) .
\end{aligned}
$$

After some calculations we have

$$
\begin{aligned}
\sin ^{2} \theta g_{N}\left(\left(\nabla F_{*}\right)(U, V), F_{*} X\right)= & g_{M}\left(\nabla_{U} \omega \psi V, X\right)-g_{M}\left(\mathcal{T}_{U} \omega V, \mathcal{B} X\right) \\
& -g_{M}\left(\mathcal{H} \nabla_{U} \omega V, \mathcal{C} X\right) .
\end{aligned}
$$

In a similar way, we get

$$
\begin{aligned}
\sin ^{2} \theta g_{N}\left(\left(\nabla F_{*}\right)(Z, U), F_{*} X\right)= & g_{M}\left(\nabla_{Z} \omega \psi U, X\right)-g_{M}\left(\mathcal{A}_{Z} \omega U, \mathcal{B} X\right) \\
& -g_{M}\left(\mathcal{H} \nabla_{Z} \omega U, \mathcal{C} X\right) .
\end{aligned}
$$


Then the proof follows from (4.24) and (4.25).

Now we establish a sharp inequality between squared mean curvature $\|H\|^{2}$ and the scalar curvature $\hat{\tau}$ of fibre through $p \in M^{5}(c)$.

Theorem 8. Let $F$ be a proper slant Riemannian submersion from a cosymplectic space form $M^{5}(c)$ onto a Riemannian manifold $\left(N^{2}, g_{N}\right)$. Then, we have

$$
\|H\|^{2} \geq \frac{8}{9}\left(\hat{\tau}-\frac{c}{4}\left(1+3 \cos ^{2} \theta\right)\right)
$$

where $H$ denotes the mean curvature of fibers. Moreover, the equality sign of (4.26) holds at a point $p$ of a fiber if and only if with respect to some suitable slant orthonormal frame $\left\{e_{1}, e_{2}=\sec \theta \psi e_{1}, e_{3}=\xi, e_{4}=\csc \theta w e_{1}, e_{5}=\right.$ $\left.\csc \theta w e_{2}\right\}$ at $p$,

$$
T_{11}^{4}=3 T_{22}^{4}, T_{12}^{4}=0 \text { and } T_{11}^{5}=0,
$$

where $T_{i j}^{\alpha}=g\left(\mathcal{T}\left(e_{i}, e_{j}\right), e_{\alpha}\right)$ for $1 \leq i, j \leq 3$ and $\alpha=4,5$.

Proof. By Corollary 1 and Lemma 6, we construct a slant orthonormal frame $\left\{e_{1}, e_{2}, e_{3}, e_{4}, e_{5}\right\}$ defined by

$$
e_{1}, e_{2}=\sec \theta \psi e_{1}, e_{3}=\xi, e_{4}=\csc \theta w e_{1}, e_{5}=\csc \theta w e_{2},
$$

where $e_{1}, e_{2}, e_{3}=\xi \in \Gamma\left(\operatorname{ker}\left(F_{*}\right)\right)$ and $e_{4}, e_{5} \in \Gamma\left(\operatorname{ker}\left(F_{*}\right)\right)^{\perp}$.

Let $\hat{\tau}$ be scalar curvature of a fibre $F^{-1}(q)$. We choose arbitrary point $p$ of the fibre $F^{-1}(q)$. We obtain

$$
\hat{\tau}(p)=\hat{K}\left(e_{1} \wedge e_{2}\right)+\hat{K}\left(e_{1} \wedge e_{3}\right)+\hat{K}\left(e_{2} \wedge e_{3}\right) .
$$

By (2.12), (2.13) and (3.6), we get

$$
\hat{K}\left(e_{1} \wedge e_{2}\right)=\frac{c}{4}\left(1+3 \cos ^{2} \theta\right)+T_{11}^{4} T_{22}^{4}+T_{11}^{5} T_{22}^{5}-\left(T_{12}^{4}\right)^{2}-\left(T_{12}^{5}\right)^{2},
$$

where $T_{i j}^{\alpha}=g\left(\mathcal{T}\left(e_{i}, e_{j}\right), e_{\alpha}\right)$ for $1 \leq i, j \leq 3$ and $\alpha=4,5$. Using Theorem 2 and the relation $(4.12)$, one has

$$
\psi e_{2}=-\cos \theta e_{1} \text { and } \omega e_{2}=\sin \theta e_{5} .
$$

From (4.7), we have

$$
g\left(\left(\nabla_{e_{2}} \psi\right) e_{2}, e_{1}\right)-g\left(\mathcal{B} \mathcal{T}_{e_{2}} e_{2}, e_{1}\right)+g\left(\mathcal{T}_{e_{2}} \omega e_{2}, e_{1}\right)=0
$$

Using (4.1), (4.2), (4.9), (4.27) and (4.30) in the last relation, we obtain

$$
\begin{aligned}
0= & g\left(\hat{\nabla}_{e_{2}} \psi e_{2}-\psi \hat{\nabla}_{e_{2}} e_{2}, e_{1}\right)-g\left(\phi \mathcal{T}_{e_{2}} e_{2}, e_{1}\right)+\sin \theta g\left(\mathcal{T}_{e_{2}} e_{5}, e_{1}\right) \\
= & -\cos \theta g\left(\hat{\nabla}_{e_{2}} e_{1}, e_{1}\right)+\cos \theta g\left(\hat{\nabla}_{e_{2}} e_{2}, e_{2}\right) \\
& +g\left(\mathcal{T}_{e_{2}} e_{2}, \psi e_{1}+\omega e_{1}\right)+\sin \theta g\left(\mathcal{T}_{e_{2}} e_{5}, e_{1}\right) \\
= & \sin \theta\left[g\left(\mathcal{T}_{e_{2}} e_{2}, e_{4}\right)-g\left(\mathcal{T}_{e_{2}} e_{1}, e_{5}\right)\right] .
\end{aligned}
$$

Since the submersion is proper, the equation (4.31) implies that

$$
T_{22}^{4}=T_{12}^{5} \text {. }
$$


Now we choose the unit normal vector $e_{4} \in \Gamma\left(\operatorname{ker}\left(F_{*}\right)\right)^{\perp}$ parallel to the mean curvature vector $H(p)$ of fibre. Then we have

$$
\|H(p)\|^{2}=\frac{1}{9}\left(T_{11}^{4}+T_{22}^{4}\right)^{2}, \quad T_{11}^{5}+T_{22}^{5}=0 .
$$

So the relation (4.29) becomes

$$
\hat{K}\left(e_{1} \wedge e_{2}\right)=\frac{c}{4}\left(1+3 \cos ^{2} \theta\right)+T_{11}^{4} T_{22}^{4}-\left(T_{11}^{5}\right)^{2}-\left(T_{12}^{4}\right)^{2}-\left(T_{22}^{4}\right)^{2} .
$$

From the trivial inequality $(\mu-3 \lambda)^{2} \geq 0$, one has $(\mu+\lambda)^{2} \geq 8\left(\lambda \mu-\lambda^{2}\right)$. Putting $\mu=T_{11}^{4}$ and $\lambda=T_{22}^{4}$ in the last inequality we find

$$
\|H\|^{2} \geq \frac{8}{9}\left[\hat{K}\left(e_{1} \wedge e_{2}\right)-\frac{c}{4}\left(1+3 \cos ^{2} \theta\right)\right] .
$$

Using (2.13) we get

$$
\hat{K}\left(e_{1} \wedge e_{3}\right)=\hat{K}\left(e_{2} \wedge e_{3}\right)=0 .
$$

By (2.13), (4.28) and the last relation we get required inequality. Moreover, the equality sign of (4.26) holds at a point $p$ of a fiber if and only if $T_{11}^{4}=3 T_{22}^{4}$, $T_{12}^{4}=0$ and $T_{11}^{5}=0$.

\subsection{Slant Riemannian submersions admitting horizontal structure vector field}

In this subsection, we will investigate the properties of slant Riemannian submersions from a cosymplectic manifold onto a Riemannian manifold such that characteristic vector field $\xi$ is a horizontal vector field.

By (3.1), (3.2) and (3.3) we have

(4.34) $\phi^{2} U=-U,\left(\nabla_{U} \phi\right) V=0$ and $g(\phi U, \phi V)=g(U, V), \forall U, V \in \Gamma\left(\operatorname{ker} F_{*}\right)$.

From (2.6), (2.10), (3.4) and (4.34) we obtain

$$
\text { i) } \mathcal{T}_{U} \xi=0
$$

ii) $\eta\left(\nabla_{U} V\right)=0$

iii) $\mathcal{A}_{X} \xi=0$

and

$$
\nabla_{U} V=-\phi \nabla_{U} \phi V
$$

for any $U, V \in \Gamma\left(\operatorname{ker} F_{*}\right)$ and $X\left(\Gamma\left(\operatorname{ker} F_{*}\right)^{\perp}\right)$. By the following same steps in (see: [5], [15]) we can prove the following characterization theorem:

Theorem 9. Let $F$ be a Riemannian submersion from a cosymplectic manifold $M\left(\phi, \xi, \eta, g_{M}\right)$ onto a Riemannian manifold $\left(N, g_{N}\right)$ such that $\xi \in\left(\Gamma\left(\operatorname{ker} F_{*}\right)^{\perp}\right)$. Then, $F$ is a slant Riemannian submersion if and only if there exist a constant $\lambda \in[0,1]$ such that

$$
\psi^{2}=-\lambda I .
$$

Furthermore, in such a case, if $\theta$ is the slant angle of $F$, then $\lambda=\cos ^{2} \theta$.

By virtue of Theorem 9, we get the following result: 
Lemma 8. Let $F$ be a slant Riemannian submersion from a cosymplectic manifold $M\left(\phi, \xi, \eta, g_{M}\right)$ onto a Riemannian manifold $\left(N, g_{N}\right)$ with slant angle $\theta$. Then we have the following relations:

$$
\begin{gathered}
g_{M}(\psi U, \psi V)=\cos ^{2} \theta g_{M}(U, V), \\
g_{M}(\omega U, \omega V)=\sin ^{2} \theta g_{M}(U, V),
\end{gathered}
$$

for any $U, V \in \Gamma\left(\operatorname{ker} F_{*}\right)$.

Remark 1 . Let $F$ be a slant Riemannian submersion from a cosymplectic manifold $M\left(\phi, \xi, \eta, g_{M}\right)$ onto a Riemannian manifold $\left(N, g_{N}\right)$ with slant angle $\theta$ and $\xi \in\left(\Gamma\left(\operatorname{ker} F_{*}\right)^{\perp}\right)$. Then there is a distribution $\mathcal{D} \subset\left(\Gamma\left(\operatorname{ker} F_{*}\right)^{\perp}\right)$ such that $\left(\operatorname{ker} F_{*}\right)^{\perp}=\omega\left(\operatorname{ker} F_{*}\right) \oplus \mathcal{D} \oplus\{\xi\}$.

In a similar argumentation of Lemma 5, we get:

Lemma 9. Let $F$ be a slant Riemannian submersion from a cosymplectic manifold $M\left(\phi, \xi, \eta, g_{M}\right)$ onto a Riemannian manifold $\left(N, g_{N}\right)$ with slant angle $\theta$ and $\xi \in\left(\Gamma\left(\operatorname{ker} F_{*}\right)^{\perp}\right)$. Then the distribution $\mathcal{D}$ is invariant under $\phi$.

Using same arguments with the proof of Theorem 4 and (4.15) we find:

Theorem 10. Let $F$ be a slant Riemannian submersion from a cosymplectic manifold $M\left(\phi, \xi, \eta, g_{M}\right)$ onto a Riemannian manifold $\left(N, g_{N}\right)$ with slant angle $\theta$. Then $\nabla Q=0$.

By virtue of (4.39), we can state:

Corollary 3. Let $F$ be a slant Riemannian submersion from a cosymplectic manifold $M^{2 m+1}\left(\phi, \xi, \eta, g_{M}\right)$ onto a Riemannian manifold $\left(N^{n}, g_{N}\right)$ with slant angle $\theta$ and $\xi \in\left(\Gamma\left(\operatorname{ker} F_{*}\right)^{\perp}\right)$. If $\left\{e_{1}, e_{2}, \ldots, e_{2 m-n+1}\right\}$ be a local orthonormal frame of $\left(\operatorname{ker} F_{*}\right)$, then $\left\{\csc \theta w e_{1}, \csc \theta w e_{2}, \ldots, \csc \theta w e_{2 m-n+1}\right\}$ is a local orthonormal frame of $\omega\left(\operatorname{ker} F_{*}\right)$. Moreover $\operatorname{dim}(\mathcal{D})=2(n-m-1)$.

Using similar proof to the Lemma 6, one can get the following:

Lemma 10. Let $F$ be a proper slant Riemannian submersion from a cosymplectic manifold $M^{2 m+1}\left(\phi, \xi, \eta, g_{M}\right)$ onto a Riemannian manifold $\left(N^{2 n+1}, g_{N}\right)$ with slant angle $\theta$ and $\xi \in\left(\Gamma\left(\operatorname{ker} F_{*}\right)^{\perp}\right)$. Then there exists a local orthonormal frame $\left\{e_{i}, \sec \theta \psi e_{i}: i=1, \ldots, m-n\right\}$ of $\left(\operatorname{ker} F_{*}\right)$.

Lemma 11. Let $F$ be a proper slant Riemannian submersion from a cosymplectic manifold $M^{2 m+1}\left(\phi, \xi, \eta, g_{M}\right)$ onto a Riemannian manifold $\left(N^{n}, g_{N}\right)$ with slant angle $\theta$ and $\xi \in\left(\Gamma\left(\operatorname{ker} F_{*}\right)^{\perp}\right)$. If $\omega$ is parallel, then we have

$$
\mathcal{T}_{\psi U} \psi U=-\cos ^{2} \theta \mathcal{T}_{U} U
$$

Proof. This proof works like the Kaehlerian case in ([25]).

The following result gives the sufficient condition to obtain the proper harmonic slant submersion. 
Theorem 11. Let $F$ be a proper slant Riemannian submersion from a cosymplectic manifold $M^{2 m+1}\left(\phi, \xi, \eta, g_{M}\right)$ onto a Riemannian manifold $\left(N^{2 n+1}, g_{N}\right)$ with slant angle $\theta$ and $\xi \in\left(\Gamma\left(\operatorname{ker} F_{*}\right)^{\perp}\right)$. If $\omega$ is parallel, then $F$ is a harmonic map.

Proof. By means of (2.16), we know that

$$
\left(\nabla F_{*}\right)(X, Y)=0
$$

for any horizontal vector fields $X, Y$. From (2.15), (2.17), (4.41) and Lemma 10 , we get

$$
\begin{aligned}
\tau(F) & =-\sum_{i=1}^{m-n}\left[\left(\nabla F_{*}\right)\left(e_{i}, e_{i}\right)+\left(\nabla F_{*}\right)\left(\sec \theta \psi e_{i}, \sec \theta \psi e_{i}\right)\right] \\
& =-\sum_{i=1}^{m-n}\left[F_{*}\left(\nabla_{e_{i}} e_{i}\right)+\sec ^{2} \theta F_{*}\left(\nabla_{\psi e_{i}} \psi e_{i}\right)\right],
\end{aligned}
$$

where $\left\{e_{1}, \sec \theta \psi e_{1}, e_{2}, \sec \theta \psi e_{2}, \ldots, e_{m-n}, \sec \theta \psi e_{m-n}\right\}$ is an orthonormal frame of $\left(\operatorname{ker} F_{*}\right)$. By applying $(2.5)$ to $(4.42)$, we obtain

$$
\tau(F)=-\sum_{i=1}^{m-n} F_{*}\left(\mathcal{T}_{e_{i}} e_{i}+\sec ^{2} \theta \mathcal{T}_{\psi e_{i}} \psi e_{i}\right) .
$$

Then using Lemma 11, we have

$$
\tau(F)=-\sum_{i=1}^{m-n} F_{*}\left(\mathcal{T}_{e_{i}} e_{i}-\mathcal{T}_{e_{i}} e_{i}\right)=0
$$

which says that $F$ is a harmonic map.

Now we give information about the geometry of the leaves of the distributions $\left(\operatorname{ker} F_{*}\right)$ and $\left(\operatorname{ker} F_{*}\right)^{\perp}$. By using the relations (4.35), (4.36) and Lemma 11 and following same way for the proof of the slant submersions from almost Hermitian manifolds (see [25]), we have:

Theorem 12. Let $F$ be a proper slant Riemannian submersion from a cosymplectic manifold $M\left(\phi, \xi, \eta, g_{M}\right)$ onto a Riemannian manifold $\left(N, g_{N}\right)$. Then the distribution (ker $\left.F_{*}\right)^{\perp}$ defines a totally geodesic foliation on $M$ if and only if

$$
g_{M}\left(\mathcal{H} \nabla_{X} Y, \omega \psi U\right)=g_{M}\left(\mathcal{A}_{X} \mathcal{B} Y, \omega U\right)+g_{M}\left(\mathcal{H} \nabla_{X} \mathcal{C} Y, \omega U\right)
$$

for any $X, Y \in \Gamma\left(\left(\operatorname{ker} F_{*}\right)^{\perp}\right)$ and $U \in \Gamma\left(\operatorname{ker} F_{*}\right)$.

Theorem 13. Let $F$ be a proper slant Riemannian submersion from a cosymplectic manifold $M\left(\phi, \xi, \eta, g_{M}\right)$ onto a Riemannian manifold $\left(N, g_{N}\right)$. Then the distribution ( $\operatorname{ker} F_{*}$ ) defines a totally geodesic foliation on $M$ if and only if

$$
g_{M}\left(\mathcal{H} \nabla_{U} \omega \psi V, X\right)=g_{M}\left(\mathcal{T}_{U} \omega V, \mathcal{B} X\right)+g_{M}\left(\mathcal{H} \nabla_{U} \omega V, \mathcal{C} X\right)
$$

for any $U, V \in \Gamma\left(\operatorname{ker} F_{*}\right)$ and $X \in \Gamma\left(\left(\operatorname{ker} F_{*}\right)^{\perp}\right)$.

From Theorem 12 and Theorem 13, we obtain: 
Corollary 4. Let $F$ be a proper slant Riemannian submersion from a cosymplectic manifold $M\left(\phi, \xi, \eta, g_{M}\right)$ onto a Riemannian manifold $\left(N, g_{N}\right)$. Then $M$ is a locally product Riemannian manifold if and only if

$$
g_{M}\left(\mathcal{H} \nabla_{X} Y, \omega \psi U\right)=g_{M}\left(\mathcal{A}_{X} \mathcal{B} Y, \omega U\right)+g_{M}\left(\mathcal{H} \nabla_{X} \mathcal{C} Y, \omega U\right)
$$

and

$$
g_{M}\left(\mathcal{H} \nabla_{U} \omega \psi V, X\right)=g_{M}\left(\mathcal{T}_{U} \omega V, \mathcal{B} X\right)+g_{M}\left(\mathcal{H} \nabla_{U} \omega V, \mathcal{C} X\right)
$$

for any $U, V \in \Gamma\left(\operatorname{ker} F_{*}\right)$ and $X \in \Gamma\left(\left(\operatorname{ker} F_{*}\right)^{\perp}\right)$.

Now we give the sufficient and necessary totally geodesic condition for a proper slant submersion $F$ from cosymplectic manifolds with $\xi \in\left(\Gamma\left(\operatorname{ker} F_{*}\right)^{\perp}\right)$.

Theorem 14. Let $F$ be a proper slant Riemannian submersion from a cosymplectic manifold $M\left(\phi, \xi, \eta, g_{M}\right)$ onto a Riemannian manifold $\left(N, g_{N}\right)$ with slant angle $\theta$ and $\xi \in\left(\Gamma\left(\operatorname{ker} F_{*}\right)^{\perp}\right)$. Then $F$ is a totally geodesic map if and only if

$$
g_{M}\left(\mathcal{H} \nabla_{U} \omega \psi V, X\right)=g_{M}\left(\mathcal{T}_{U} \omega V, \mathcal{B} X\right)+g_{M}\left(\mathcal{H} \nabla_{U} \omega V, \mathcal{C} X\right)
$$

and

$$
g_{M}\left(\mathcal{H} \nabla_{Y} \omega \psi U, X\right)=g_{M}\left(\mathcal{A}_{Y} \omega U, \mathcal{B} X\right)+g_{M}\left(\mathcal{H} \nabla_{Y} \omega U, \mathcal{C} X\right)
$$

for $U, V \in \Gamma\left(\operatorname{ker} F_{*}\right)$ and $X, Y \in \Gamma\left(\left(\operatorname{ker} F_{*}\right)^{\perp}\right)$.

Proof. Using (2.16), we have

$$
\left(\nabla F_{*}\right)(X, Y)=0
$$

for $X, Y \in \Gamma\left(\left(\operatorname{ker} F_{*}\right)^{\perp}\right)$. Thus it is enough to prove that $\left(\nabla F_{*}\right)(U, V)=0$ and $\left(\nabla F_{*}\right)(X, U)=0$ for $U, V \in \Gamma\left(\operatorname{ker} F_{*}\right)$ and $X \in \Gamma\left(\left(\operatorname{ker} F_{*}\right)^{\perp}\right)$. From (4.1) and (4.35) we obtain

$$
g_{N}\left(\left(\nabla F_{*}\right)(U, V), F_{*} X\right)=g_{M}\left(\nabla_{U} \phi \psi V, X\right)-g_{M}\left(\nabla_{U} \omega V, \phi X\right) .
$$

Using again (4.1) and (4.2), we get

$$
\begin{aligned}
g_{N}\left(\left(\nabla F_{*}\right)(U, V), F_{*} X\right)= & g_{M}\left(\nabla_{U} \psi^{2} V, X\right)+g_{M}\left(\nabla_{U} \omega \psi V, X\right) \\
& -g_{M}\left(\nabla_{U} \omega V, \mathcal{B} X\right)-g_{M}\left(\nabla_{U} \omega V, \mathcal{C} X\right) .
\end{aligned}
$$

By Theorem 9, (2.5), (2.6) and (4.35), we obtain

$$
\begin{aligned}
g_{N}\left(\left(\nabla F_{*}\right)(U, V), F_{*} X\right)= & -\cos ^{2} \theta g_{M}\left(\nabla_{U} V, X\right)+g_{M}\left(\nabla_{U} \omega \psi V, X\right) \\
& -g_{M}\left(\mathcal{T}_{U} \omega V, \mathcal{B} X\right)-g_{M}\left(\mathcal{H} \nabla_{U} \omega V, \mathcal{C} X\right) .
\end{aligned}
$$

By simple calculations, we have

$$
\begin{aligned}
\sin ^{2} \theta g_{N}\left(\left(\nabla F_{*}\right)(U, V), F_{*} X\right)= & g_{M}\left(\nabla_{U} \omega \psi V, X\right)-g_{M}\left(\mathcal{T}_{U} \omega V, \mathcal{B} X\right) \\
& -g_{M}\left(\mathcal{H} \nabla_{U} \omega V, \mathcal{C} X\right) .
\end{aligned}
$$

In a similar way, we get

$$
\begin{aligned}
\sin ^{2} \theta g_{N}\left(\left(\nabla F_{*}\right)(Y, U), F_{*} X\right)= & g_{M}\left(\nabla_{Y} \omega \psi U, X\right)-g_{M}\left(\mathcal{A}_{Y} \omega U, \mathcal{B} X\right) \\
& -g_{M}\left(\mathcal{H} \nabla_{Y} \omega U, \mathcal{C} X\right) .
\end{aligned}
$$

Combining (4.43) and (4.44), we get requested equations. 
Now we give a sharp inequality between squared mean curvature $\|H\|^{2}$ and the scalar curvature $\hat{\tau}$ of fibre through $p \in M^{5}(c)$ such that characteristic vector field $\xi$ is horizontal.

Theorem 15. Let $F$ be a proper slant Riemannian submersion from a cosymplectic space form $M^{5}(c)$ onto a Riemannian manifold $\left(N^{3}, g_{N}\right)$. Then, we have

$$
\|H\|^{2} \geq\left(\hat{\tau}-\frac{c}{4}\left(1+3 \cos ^{2} \theta\right)\right) .
$$

Moreover, the equality sign of (4.45) holds at a point $p$ of a fiber if and only if with respect to some suitable slant orthonormal frame $\left\{e_{1}, e_{2}=\sec \theta \psi e_{1}\right.$, $\left.e_{3}=\csc \theta w e_{1}, e_{4}=\csc \theta w e_{2}, e_{5}=\xi\right\}$ at $p$,

$$
T_{11}^{4}=-T_{22}^{4} \text {, and } T_{11}^{3}=T_{12}^{3}=T_{22}^{3}=0=T_{i j}^{5},
$$

where $T_{i j}^{\alpha}=g\left(\mathcal{T}\left(e_{i}, e_{j}\right), e_{\alpha}\right)$ for $1 \leq i, j \leq 2$ and $3 \leq \alpha \leq 5$. Here $H$ is the mean curvature of fiber.

Proof. By Corollary 3 and Lemma 10, we construct a slant orthonormal frame $\left\{e_{1}, e_{2}, e_{3}, e_{4}, e_{5}\right\}$ defined by

$$
e_{1}, e_{2}=\sec \theta \psi e_{1}, e_{3}=\csc \theta w e_{1}, e_{4}=\csc \theta w e_{2}, e_{5}=\xi,
$$

where $e_{1}, e_{2} \in \Gamma\left(\operatorname{ker}\left(F_{*}\right)\right)$ and $e_{3}, e_{4}, e_{5}=\xi \in \Gamma\left(\operatorname{ker}\left(F_{*}\right)\right)^{\perp}$.

Let $\hat{\tau}$ be scalar curvature of a fibre $F^{-1}(q)$. We choose an arbitrary point $p$ of the fibre $F^{-1}(q)$. Since $\operatorname{dim}\left(\operatorname{Ker} F_{*}\right)=2$, we obtain

$$
\hat{\tau}(p)=\hat{K}\left(e_{1} \wedge e_{2}\right) .
$$

By (2.12), (2.13), (4.35(i)) and (3.6), we get

$$
\hat{K}\left(e_{1} \wedge e_{2}\right)=\frac{c}{4}\left(1+3 \cos ^{2} \theta\right)+T_{11}^{3} T_{22}^{3}+T_{11}^{4} T_{22}^{4}-\left(T_{12}^{3}\right)^{2}-\left(T_{12}^{4}\right)^{2},
$$

where $T_{i j}^{\alpha}=g\left(\mathcal{T}\left(e_{i}, e_{j}\right), e_{\alpha}\right)$ for $1 \leq i, j \leq 2$ and $\alpha=3,4,5$. Using Theorem 9 and the relation $(4.39)$, one has

$$
\psi e_{2}=-\cos \theta e_{1} \quad \text { and } \quad \omega e_{2}=\sin \theta e_{5} .
$$

From (4.7) we have

$$
g\left(\left(\nabla_{e_{2}} \psi\right) e_{2}, e_{1}\right)=g\left(\mathcal{B} \mathcal{T}_{e_{2}} e_{2}, e_{1}\right)-g\left(\mathcal{T}_{e_{2}} \omega e_{2}, e_{1}\right) .
$$

Using (4.1), (4.2), (4.9), (4.46) and (4.49) in the last relation, we obtain

$$
\begin{aligned}
0= & g\left(\hat{\nabla}_{e_{2}} \psi e_{2}-\psi \hat{\nabla}_{e_{2}} e_{2}, e_{1}\right)-g\left(\phi \mathcal{T}_{e_{2}} e_{2}, e_{1}\right)+\sin \theta g\left(\mathcal{T}_{e_{2}} e_{4}, e_{1}\right) \\
= & -\cos \theta g\left(\hat{\nabla}_{e_{2}} e_{1}, e_{1}\right)+\cos \theta g\left(\hat{\nabla}_{e_{2}} e_{2}, e_{2}\right) \\
& +g\left(\mathcal{T}_{e_{2}} e_{2}, \psi e_{1}+\omega e_{1}\right)+\sin \theta g\left(\mathcal{T}_{e_{2}} e_{4}, e_{1}\right) \\
= & \sin \theta\left[g\left(\mathcal{T}_{e_{2}} e_{2}, e_{3}\right)-g\left(\mathcal{T}_{e_{2}} e_{1}, e_{4}\right)\right] .
\end{aligned}
$$

Since our submersion is proper, the equation (4.50) implies

$$
T_{22}^{3}=T_{12}^{4} \text {. }
$$


Because of $\mathcal{T}_{e_{\alpha}} \xi=0$, we can choose the unit normal vector $e_{4} \in \Gamma\left(\operatorname{ker}\left(F_{*}\right)\right)^{\perp}$ parallel to the mean curvature vector $H(p)$ of fibre. Then we have

$$
\|H(p)\|^{2}=\frac{1}{4}\left(T_{11}^{4}+T_{22}^{4}\right)^{2}, \quad T_{11}^{3}+T_{22}^{3}=0 .
$$

So the relation $(4.48)$ becomes

$$
\hat{K}\left(e_{1} \wedge e_{2}\right)=\frac{c}{4}\left(1+3 \cos ^{2} \theta\right)+T_{11}^{4} T_{22}^{4}-\left(T_{11}^{3}\right)^{2}-\left(T_{12}^{3}\right)^{2}-\left(T_{22}^{3}\right)^{2} .
$$

From the trivial inequality $(\mu-\lambda)^{2} \geq 0$, one has $(\mu+\lambda)^{2} \geq 4 \lambda \mu$. Putting $\mu=T_{11}^{4}$ and $\lambda=T_{22}^{4}$ in the last inequality, we find

$$
\|H\|^{2} \geq\left[\hat{K}\left(e_{1} \wedge e_{2}\right)-\frac{c}{4}\left(1+3 \cos ^{2} \theta\right)\right]
$$

By (4.47) and the last relation, we get required inequality. Moreover, the equality sign of (4.45) holds at a point $p$ of a fiber if and only if $T_{11}^{4}=-T_{22}^{4}$, $T_{11}^{3}=T_{12}^{3}=T_{22}^{3}=0=T_{i j}^{5}$.

Recently H. Tastan, [28], proved that the horizontal distribution of a Lagrangian submersion from a Kaehlerian manifold to a Riemannian manifold is integrable and totally geodesic. He also showed that such a submersion is totally geodesic if and only if it has totally geodesic fibres.

Anti-invariant submersions are special slant submersions with slant angle $\theta=\frac{\pi}{4}$. Now we focus on anti-invariant submersions from a cosymplectic manifold to a Riemannian manifold such that $\left(\operatorname{ker} F_{*}\right)^{\perp}=\phi\left(\operatorname{ker}\left(F_{*}\right)\right) \oplus\{\xi\}$. In this case we note that $\operatorname{ker}\left(F_{*}\right)=\phi\left(\left(\operatorname{ker} F_{*}\right)^{\perp}\right)$. The authors investigated such a submersions in [17]. We will give some additional results.

By means of (4.34) and (2.5)-(2.8), we give:

Lemma 12. Let $F$ be a Riemannian submersion from a cosymplectic manifold $M\left(\phi, \xi, \eta, g_{M}\right)$ onto a Riemannian manifold $\left(N, g_{N}\right)$ such that $\left(\operatorname{ker} F_{*}\right)^{\perp}=$ $\phi\left(\operatorname{ker}\left(F_{*}\right)\right) \oplus\{\xi\}$. Then

$$
\text { i) } \mathcal{T}_{U} \phi E=\phi \mathcal{T}_{U} E \text { and ii) } \mathcal{A}_{X} \phi E=\phi \mathcal{A}_{X} E
$$

for any $U \in \Gamma\left(\operatorname{ker} F_{*}\right), X \in \Gamma\left(\left(\operatorname{ker} F_{*}\right)^{\perp}\right)$ and $E \in \Gamma(T M)$.

By (2.4) and (4.53), we obtain the following result.

Corollary 5. Let $F$ be a Riemannian submersion from a cosymplectic manifold $M\left(\phi, \xi, \eta, g_{M}\right)$ onto a Riemannian manifold $\left(N, g_{N}\right)$ such that (ker $\left.F_{*}\right)^{\perp}$ $=\phi\left(\operatorname{ker}\left(F_{*}\right)\right) \oplus\{\xi\}$. Then, for any $X, Y \in \Gamma\left(\left(\operatorname{ker} F_{*}\right)^{\perp}\right)$, we have

$$
\mathcal{A}_{X} \phi Y=-\mathcal{A}_{Y} \phi X \text {. }
$$

Theorem 16. Let $F$ be a Riemannian submersion from a cosymplectic manifold $M\left(\phi, \xi, \eta, g_{M}\right)$ onto a Riemannian manifold $\left(N, g_{N}\right)$ such that (ker $\left.F_{*}\right)^{\perp}=$ $\phi\left(\operatorname{ker}\left(F_{*}\right)\right) \oplus\{\xi\}$. Then the horizontal distribution (ker $\left.F_{*}\right)^{\perp}$ is integrable and totally geodesic. 
Proof. Since the tensor field is $\mathcal{A}=\mathcal{A}_{H}$, it is sufficient to show that $\mathcal{A}_{X}=0$ for any $X \in \Gamma\left(\left(\operatorname{ker} F_{*}\right)^{\perp}\right)$. If $Y$ and $Z$ are horizontal vector fields on $M$, we have

$$
\begin{gathered}
g_{M}\left(\mathcal{A}_{X} \phi Y, Z\right) \stackrel{(4.54)}{=}-g_{M}\left(\mathcal{A}_{Y} \phi X, Z\right) \stackrel{(4.53)}{=}-g_{M}\left(\phi \mathcal{A}_{Y} X, Z\right) \\
\phi \stackrel{\text { anti-sym }}{=} g_{M}\left(\mathcal{A}_{Y} X, \phi Z\right) \stackrel{(2.4)}{=}-g_{M}\left(\mathcal{A}_{X} Y, \phi Z\right) \stackrel{(2.11)}{=} g_{M}\left(\mathcal{A}_{X} \phi Z, Y\right) \\
\stackrel{(4.54)}{=}-g_{M}\left(\mathcal{A}_{Z} \phi X, Y\right) \stackrel{(2.11)}{=} g_{M}\left(\mathcal{A}_{Z} Y, \phi X\right) \stackrel{(2.4)}{=}-g_{M}\left(\mathcal{A}_{Y} Z, \phi X\right) \\
\stackrel{(2.11)}{=} g_{M}\left(\mathcal{A}_{Y} \phi X, Z\right) \stackrel{(4.54)}{=}-g_{M}\left(\mathcal{A}_{X} \phi Y, Z\right) .
\end{gathered}
$$

So we get

$$
\mathcal{A}_{X} \phi Y=0
$$

which implies $\phi \mathcal{A}_{X} Y=0$. By (3.1) we obtain

$$
\mathcal{A}_{X} Y=-g_{M}\left(\mathcal{A}_{X} Y, \xi\right) \xi=g_{M}\left(\mathcal{A}_{X} \xi, Y\right) \xi=0 .
$$

Since $U$ is a vertical vector field on $M, \phi U$ will be a horizontal vector field on $M$. Therefore we obtain $\mathcal{A}_{X} \phi U=0$. Using (3.1) and (4.53), we have

$$
\mathcal{A}_{X} U=0 \text {. }
$$

By virtue of (4.56) and (4.57) we get $\mathcal{A}_{X}=0$. The fact that (ker $\left.F_{*}\right)^{\perp}$ is totally geodesic is obvious from (4.55) and (see [17], Corollary 5).

Using Theorem 16 and (see [17], Corollary 6 and Theorem 15), we obtain the following theorem.

Theorem 17. Let $F$ be a Riemannian submersion from a cosymplectic manifold $M\left(\phi, \xi, \eta, g_{M}\right)$ onto a Riemannian manifold $\left(N, g_{N}\right)$ such that $\left(\operatorname{ker} F_{*}\right)^{\perp}=$ $\phi\left(\operatorname{ker}\left(F_{*}\right)\right) \oplus\{\xi\}$. Then $F$ is a totally geodesic map if and only if it has totally geodesic fibers.

Since horizontal distribution for a slant Riemannian submersion from a cosymplectic manifold $M\left(\phi, \xi, \eta, g_{M}\right)$ onto a Riemannian manifold $\left(N, g_{N}\right)$ with $\left(\operatorname{ker} F_{*}\right)^{\perp}=\phi\left(\operatorname{ker}\left(F_{*}\right)\right) \oplus\{\xi\}$ is integrable, the equation (2.14) reduces to

$$
R(Y, W, V, X)=g_{M}\left(\left(\nabla_{X} \mathcal{T}\right)(V, W), Y\right)-g_{M}\left(\mathcal{T}_{V} X, \mathcal{T}_{W} Y\right)
$$

for any $X, Y, Z \in \Gamma\left(\left(\operatorname{ker} F_{*}\right)^{\perp}\right), V, W \in \Gamma\left(\operatorname{ker} F_{*}\right)$.

From (3.5) and (4.58) we give:

Theorem 18. Let $F$ be a Riemannian submersion from a cosymplectic manifold $M\left(\phi, \xi, \eta, g_{M}\right)$ onto a Riemannian manifold $\left(N, g_{N}\right)$ such that $\left(\operatorname{ker} F_{*}\right)^{\perp}$ $=\phi\left(\operatorname{ker}\left(F_{*}\right)\right) \oplus\{\xi\}$. Then the $\phi$-sectional curvature $H$ of $M$ satisfies

$$
\begin{aligned}
& H(V)=g_{M}\left(\left(\nabla_{\phi V} \mathcal{T}\right)(V, V), \phi V\right)-g_{M}\left(\mathcal{T}_{V} \phi V, \mathcal{T}_{V} \phi V\right), \\
& H(X)=g_{M}\left(\left(\nabla_{X} \mathcal{T}\right)(\phi X, \phi X), X\right)-g_{M}\left(\mathcal{T}_{\phi X} X, \mathcal{T}_{\phi X} X\right),
\end{aligned}
$$

for any $X, Y, Z \in \Gamma\left(\left(\operatorname{ker} F_{*}\right)^{\perp}\right), V, W \in \Gamma\left(\operatorname{ker} F_{*}\right)$. 
It is well known from [8] that if the tensor field $\mathcal{T}$ is parallel, i.e., $\nabla \mathcal{T}=0$ for a Riemannian submersion, then $\mathcal{T}=0$. From Theorem 18, we have:

Corollary 6. Let $F$ be a Riemannian submersion from a cosymplectic manifold $M\left(\phi, \xi, \eta, g_{M}\right)$ onto a Riemannian manifold $\left(N, g_{N}\right)$ such that $\left(\operatorname{ker} F_{*}\right)^{\perp}=$ $\phi\left(\operatorname{ker}\left(F_{*}\right)\right) \oplus\{\xi\}$. If the tensor field $\mathcal{T}$ is parallel, then the $\phi$-sectional curvature $H$ of $M$ vanishes.

Corollary 7. Let $M(c \neq 0)$ be a cosymplectic space form. Then there is no Riemannian submersion $F$ with totally geodesic fibres from a cosymplectic space form $M(c \neq 0)$ onto a Riemannian manifold $\left(N, g_{N}\right)$ such that $\left(\operatorname{ker} F_{*}\right)^{\perp}$ $=\phi\left(\operatorname{ker}\left(F_{*}\right)\right) \oplus\{\xi\}$.

Acknowledgement. The authors are grateful to the referee for the valuable suggestions and comments towards the improvement of the paper.

\section{References}

[1] P. Baird and J. C. Wood, Harmonic Morphisms Between Riemannian Manifolds, London Mathematical Society Monographs, 29, Oxford University Press, The Clarendon Press, Oxford, 2003.

[2] D. E. Blair, Contact Manifolds in Riemannian Geometry, Lectures Notes in Mathematics 509, Springer-Verlag, Berlin, 1976.

[3] J. P. Bourguignon and H. B. Lawson, Stability and isolation phenomena for Yang-mills fields, Comm. Math. Phys. 79 (1981), no. 2, 189-230.

[4] - Mathematician's visit to Kaluza-Klein theory, Rend. Sem. Mat. Univ. Politec. Torino (1989), Special Issue, 143-163.

[5] J. L. Cabrerizo, A. Carriazo, L. M. Fernandez, and M. Fernandez, Slant submanifolds in Sasakian Manifolds, Glasg. Math. J. 42 (2000), no. 1, 125-138.

[6] B. Y. Chen, Geometry of Slant Submanifolds, Katholieke Universiteit Leuven, Leuven, 1990.

[7] D. Chinea, Almost contact metric submersions, Rend. Circ. Mat. Palermo (2) 34 (1985), no. $1,89-104$

[8] R. H. Escobales Jr., Riemannian submersions with totally geodesic fibers, J. Differential Geom. 10 (1975), 253-276.

[9] A. Gray, Pseudo-Riemannian almost product manifolds and submersions, J. Math. Mech. 16 (1967), 715-737.

[10] S. Ianus, A. M. Ionescu, R. Mazzocco, and G. E. Vilcu, Riemannian submersions from almost contact metric manifolds, arXiv: 1102.1570v1 [math. DG].

[11] S. Ianus, R. Mazzocco, and G. E. Vilcu, Riemannian submersions from quaternionic manifolds, Acta Appl. Math. 104 (2008), no. 1, 83-89.

[12] S. Ianus and M. Visinescu, Kaluza-Klein theory with scalar fields and generalized Hopf manifolds, Class. Quantum Gravity 4 (1987), 1317-1325.

[13] Space-time compactification and Riemannian submersions, In: Rassias, G.(ed.) The Mathematical Heritage of C. F. Gauss, (1991), 358-371, World Scientific, River Edge.

[14] B. H. Kim, Fibred Riemannian spaces with quasi Sasakian structure, Hiroshima Math. J. 20 (1990), no. 3, 477-513.

[15] I. Kupeli Erken and C. Murathan, Slant Riemannian submersions from Sasakian manifolds, arXiv: 1309.2487v1 [math. DG].

[16] G. D. Ludden, Submanifolds of cosymplectic manifolds, J. Differential Geom. 4 (1970), $237-244$. 
[17] C. Murathan and I. Kupeli Erken, Anti-invariant Riemannian submersions from cosymplectic manifolds, arXiv:1302.5108v1 [math. DG].

[18] M. T. Mustafa, Applications of harmonic morphisms to gravity, J. Math. Phys. 41 (2000), no. 10, 6918-6929

[19] Z. Olszak, On almost cosymplectic manifolds, Kodai Math. J. 4 (1981), no. 2, 239-250.

[20] B. O'Neill, The fundamental equations of a submersion, Michigan Math. J. 13 (1966), 459-469.

[21] _ Semi-Riemannian Geometry with Applications to Relativity, Academic Press, New York-London 1983.

[22] K. S. Park, H-slant submersions, Bull. Korean Math. Soc. 49 (2012), no. 2, 329-338.

[23] — H-semi-invariant submersions, Taiwanese J. Math. 16 (2012), no. 5, 1865-1878.

[24] B. Sahin, Anti-invariant Riemannian submersions from almost Hermitian manifolds, Cent. Eur. J. Math. 8 (2010), no. 3, 437-447.

[25] _ Slant submersions from almost Hermitian manifolds, Bull. Math. Soc. Sci. Math. Roumanie (N.S) 54(102) (2011), no. 1, 93-105.

[26] _ Riemannian submersions from almost Hermitian manifolds, Taiwanese J. Math. 17 (2013), no. 2, 629-659.

[27] - Semi-invariant submersions from almost Hermitian manifolds, Canad. Math. Bull. 56 (2013), no. 1, 173-183.

[28] H. M. Taştan, On Lagrangian submersion, arXiv: 1311.1676v1 [math. DG].

[29] B. Watson, Almost Hermitian submersions, J. Differential Geom. 11 (1976), no. 1, 147165.

[30] $, G, G^{\prime}$-Riemannian submersions and nonlinear gauge field equations of general relativity, In: Rassias, T. (ed.) Global Analysis-Analysis on manifolds, dedicated M. Morse, 324-349, Teubner-Texte Math., 57, Teubner, Leipzig, 1983.

[31] D. W. Yoon, Inequality for Ricci curvature of slant submanifolds in cosymplectic space forms, Turkish J. Math. 30 (2006), no. 1, 43-56.

İREM KÜPELI ERKEN

Department of Mathematics

FACUlTy of ART AND SCIENCE

ULUDAG UNIVERSITY

GÖRÜKLE 16059 BURSA-TURKEY

E-mail address: iremkupeli@uludag.edu.tr

Cengizhan Murathan

Department of Mathematics

FACUlty of ARt ANd SCIENCE

ULUDAG UNIVERSITY

GÖRÜKLE 16059 BuRsA-TURKEY

E-mail address: cengiz@uludag.edu.tr 